\title{
Preliminary Evaluation and Comparison of Atmospheric Turbulence Rejection Performance for Infinite and Receding Horizon Control in Adaptive Optics Systems
}

\author{
Mikhail V. Konnik ${ }^{1}$ and José De Doná ${ }^{1}$ \\ ${ }^{1}$ Faculty of Engineering and Built Environment, the University of Newcastle, Australia.
}

\begin{abstract}
Model-based optimal control such as Linear Quadratic Gaussian (LQG) control has been attracting considerable attention for adaptive optics systems. The ability of LQG to handle the complex dynamics of deformable mirrors and its relatively simple implementation makes LQG attractive for large adaptive optics systems. However, LQG has its own share of drawbacks, such as suboptimal handling of constraints on actuators movements and possible numerical problems in case of fast sampling rate discretisation of the corresponding matrices.

Unlike LQG, the Receding Horizon Control (RHC) technique provides control signals for a deformable mirror that are optimal within the prescribed constraints. This is achieved by reformulating the control problem as an online optimisation problem that is solved at each sampling instance. In the unconstrained case, RHC produces the same control signals as LQG. However, when the control signals reach the constraints of actuator's allowable movement in a deformable mirror, RHC finds the control signals that are optimal within those constraints, rather than just clipping the unconstrained optimum as commonly done in LQG control.

The article discusses the consequences of high-gain LQG control operation in the case when the constraints on the actuator's movement are reached. It is shown that clipping / saturating the control signals is not only suboptimal, but may be hazardous for the surface of a deformable mirror. The results of numerical simulations indicate that high-gain LQG control can lead to abrupt changes and spikes in the control signal when saturation occurs. The article further discusses a possible link between high-gain LQG and the waffle mode in the closed-loop operation of astronomical adaptive optics systems.
\end{abstract}

Performance evaluation of Receding Horizon Control in terms of atmospheric disturbance rejection and a comparison with Linear Quadratic Gaussian control are performed. The results of the numerical simulations suggest that the disturbance rejection performance in the unconstrained case is the same for LQG and RHC, while RHC clearly outperforms the saturated LQG control in terms of atmospheric turbulence rejection. More importantly, RHC can be used in high-gain mode, unlike LQG, providing better atmospheric disturbance rejection in the constrained case.

Keywords: constrained control, receding horizon control, quadratic programming

\section{END-TO-END SIMULATION ENVIRONMENT FOR THE PERFORMANCE EVALUATION OF INFINITE AND RECEDING HORIZON CONTROLLERS}

This section briefly outlines the simulation environment that we used for end-to-end optical closed-loop numerical experiments. All simulations were carried out using our own Adaptive Optics Real Time Analyser (AORTA), a MATLAB-based simulator of adaptive optics components. In this work we use a single-layer moving atmosphere as an output disturbance to be cancelled by Receding Horizon Control (RHC) and Infinite Horizon Control (also called Linear Quadratic Gaussian, LQG control) in the constrained and unconstrained cases. The comparison is performed using the sum of squares and the variance of the residual (uncompensated) atmospheric turbulence.

Corresponding author: Mikhail V. Konnik: mail@mvkonnik.info, or mikhail.konnik@uon.edu.au Contributing authors emails Jose.Dedona@newcastle.edu.au 


\subsection{Atmosphere modelling and system identification}

The phase screen for the atmospheric turbulence layer was generated using the von Kármán model with the power spectrum given by $\Phi_{\phi}^{v K}(\kappa)=0.49 \cdot r_{0}^{-5 / 3} \cdot\left(\kappa^{2}+\kappa_{0}^{2}\right)^{-11 / 6}$, where $\kappa$ is the spatial wave number and $\kappa_{0}=\frac{2 \pi}{L_{0}}$, where $L_{0}$ is the outer scale. The "subharmonics" method was used to generate the phase screen ${ }^{1,2} \phi(u, v)$ of size $M \times M$ that was implemented similar to Schmidt. ${ }^{3}$ In simulations we assumed a $D=4 \mathrm{~m}$ telescope aperture observing in a visible light $(\lambda=550 \mathrm{~nm})$. A square $M \times M$ phase screen $\phi(u, v)$ with $M=256$ pixels was generated assuming the Fried parameter $r_{0}=0.1$ and the outer scale $L_{0}=20 \mathrm{~m}$.

\subsubsection{Moving atmosphere model}

We use the algorithm proposed by Assémat et al. ${ }^{4}$ to simulate an infinitely long phase screen. During the simulation, the phase screen is extended by adding ("extruding") a new column $\mathbf{X}=\mathbf{A} \mathbf{Z}+\mathbf{B} \beta$ to the existing phase screen $\phi(u, v)$, where the matrix $\mathbf{A}$ of size $M \times\left(M N_{c o l}\right)$ and the matrix $\mathbf{B}$ of size $M \times M$ are defined in Assémat et al., ${ }^{4}$ the vector $\mathbf{Z}$ of size $\left(M N_{c o l}\right) \times 1$ contains the last $N_{c o l}$ columns of phase screen $\phi(u, v)$, and the vector $\beta$ of size $M \times 1$ is a Gaussian vector with zero mean and unit variance. In simulations, we use the last $N_{c o l}=2$ columns of the phase screen to compute the new phase values, which is considered adequate. ${ }^{4} \mathrm{~A}$ single layer of the turbulence is assumed to be on an altitude of $5 \mathrm{~km}$ with a wind speed of $20 \mathrm{~m} / \mathrm{s}$ if not stated otherwise.

\subsubsection{System identification of the atmospheric turbulence}

Since we use a model-based control, the state space matrices that correspond to atmospheric turbulence have to be estimated via stochastic system identification from the wavefront sensed data. Assuming the Forward Innovation Stochastic model:

$$
x_{k+1}=A_{a t m} x_{k}+K_{a t m}^{f} e_{k}, \quad y_{k}=C_{a t m} x_{k}+e_{k}
$$

we use the stochastic Algorithm 3 ("sto_pos") from Van Overschee ${ }^{5}$ that allows to get the state space matrices directly. The algorithm transforms the data into block Hankel matrices $Y_{p}=Y_{0 \mid k-1}$ and $Y_{f}=$ $Y_{k \mid 2 k-1}$ for past and future outputs, respectively. Next, the oblique projection $\mathcal{O}_{i}=Y_{f} / \mathbf{Y}_{\mathbf{p}}$ is computed. The order of the state space matrices is determined by inspecting the singular values (see Fig. 1) of the SVD of the weighted oblique projection: $W_{1} \mathcal{O}_{i} W_{2}=U S V^{T}$.

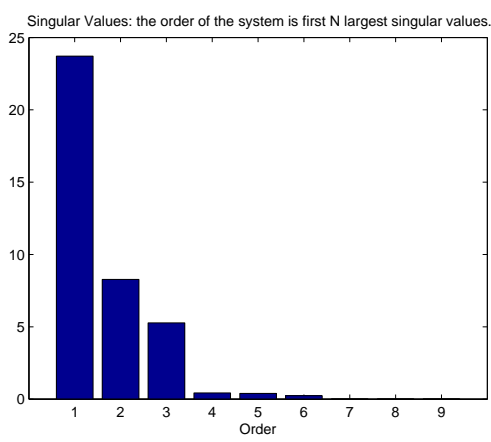

Figure 1. System identification of atmospheric turbulence model: estimating the order of the system (in this case the order is 1 for a 3 -input data).

Since we use the state space matrices obtained via stochastic system identification from limited data of the moving atmosphere model, the reported performance of the controllers is not the best possible but it fulfils practical considerations.

\subsection{Wavefront sensing and reconstruction}

The numerical model of the Shack-Hartmann wavefront sensor (WFS) uses $16 \times 16$ lenslets with $16 \times 16$ pixels per lenslet. Each lenslets' focal distance is assumed to be $10 \mathrm{~mm}$, the wavelength of observation is $550 \mathrm{~nm}$. We use WCoG centroiding algorithm ${ }^{6}$ with the parameter $\sigma_{W C o G}=6$. The model of the CCD sensor in the WFS has $5 \mu \mathrm{m}$ pixels, and no noise has been introduced. The wavefront is reconstructed using the modified Hudgin geometry, which was implemented according to Poyneer et al. ${ }^{7}$

\subsection{Surface and Dynamics models of the deformable mirror}

The model of the continuous-faceplate deformable mirror consists of the model of the surface and dynamic response of the piezoelectrical actuators. The surface is modelled via the Radial Basis Functions ${ }^{8,9}$ with a Gaussian kernel. The dynamics of piezoelectrical actuators is described via a first-order transfer function with a fast pole. 


\subsubsection{Radial Basis Functions model of the surface of a deformable mirror}

A radial basis function (RBF) is a real-valued function ${ }^{9}$ whose values depend only on the distance $\phi(r)$, where $r=$ $\left\|x-x_{i}\right\|$ is the Euclidean distance and $x_{i}$ are the centres (or knots). RBFs are typically used to construct function approximations of the form $y(x)=\sum_{i=1}^{n} \lambda_{i} \phi\left(\left\|x-x_{i}\right\|\right)$, where the approximating function $y(x)$ is represented as a sum of $n$ radial basis functions, each associated with a different centre $x_{i}$, and weighted by an appropriate weighting coefficient $\lambda_{i}$. We model the surface of the continuous-faceplate deformable mirror using the Gaussian kernel $\phi(r)=e^{-(\varepsilon r)^{2}}$ for RBF with a "scale factor" $\varepsilon$, where $\varepsilon=\frac{1}{2 \sigma^{2}}$ and $\sigma$ being a width parameter. This allows modelling a smooth continuous surface of a DM.

\subsubsection{Dynamics models of the Deformable Mirror}

The dynamics of the actuators is assumed to be a first-order transfer function (TF) with a fast pole, and the TF for the $(i, i)$-th actuator is:

$$
G(s)_{\mathrm{plant}}^{i, i}=\frac{1}{s T_{\mathcal{N}}+1},
$$

where the mean value of the response time of the actuator is $T=2 * 10^{-3} \mathrm{~s}$ was used in a Normal Distribution $T_{\mathcal{N}} \sim \mathcal{N}\left(T, \sigma_{T}^{2}\right)$ with standard deviation $\sigma_{T}=\alpha \cdot T$ to account for the different dynamics of the actuators that varies from one actuator to another. In our simulations we use $\alpha=0.05$ if not stated otherwise.

The inter-actuator coupling, which models the influence of an energised actuator upon its neighbours, can be described as a proportional relationship between the actuators' transfer functions characterised by a coupling degree $\gamma$. The transfer matrix (TM) therefore contains off-diagonal elements $G_{i, j}$ :

$$
G_{\text {plant }}^{\mathrm{TM}}(s)=\left[\begin{array}{cccccc}
\mathbf{G}_{\mathbf{1}, \mathbf{1}} & G_{1,2} & 0 & G_{1,4} & 0 & \cdots \\
G_{2,1} & \mathbf{G}_{\mathbf{2}, \mathbf{2}} & G_{2,3} & 0 & G_{2,5} & \cdots \\
\vdots & \vdots & \ddots & & &
\end{array}\right] .
$$

In this $3 \times 3$ actuators grid example, the coupling TF $G_{5,2}(s)$ between the actuator 5 and actuator 2 , for instance, is $G_{5,2}(s)=\gamma \cdot G_{5,5}(s)$, where $\gamma$ is the coupling degree.

\subsection{Model of the system dynamics and closed-loop control}

The continuous TM of the plant $G_{\text {plant }}^{\mathrm{TM}}(s)$ in $(2)-(3)$ was converted to the state space formulation and discretised with a sampling time of $T_{s}=10^{-3}$ seconds. The state space model was augmented to account for both deformable mirror and identified atmospheric turbulence dynamics in (1) as follows:

$$
\begin{array}{r}
A=\left[\begin{array}{cc}
A_{\text {plant }} & 0 \\
0 & A_{\text {atm }}
\end{array}\right], B=\left[\begin{array}{c}
B_{\text {plant }} \\
0
\end{array}\right], G=\left[\begin{array}{c}
0 \\
K_{\text {atm }}^{f}
\end{array}\right] \\
C=\left[C_{\text {plant }} C_{\text {atm }}\right] .
\end{array}
$$

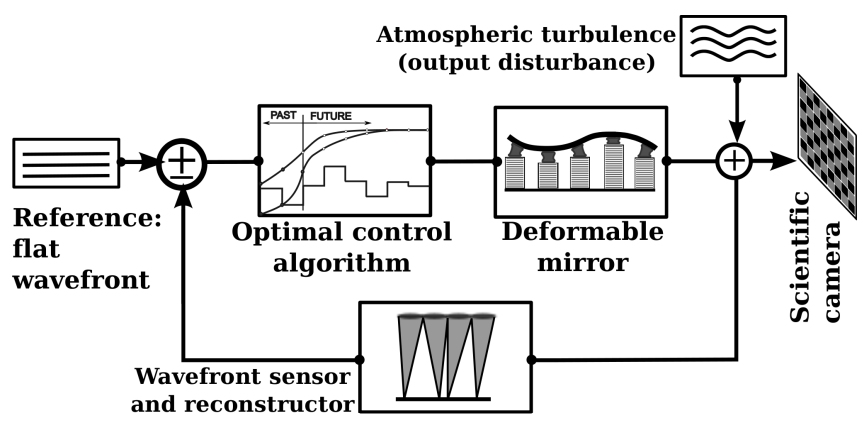

Figure 2. The overall control scheme of an AO system.

We model the adaptive optics (AO) system as a Linear Time-Invariant Multi-Input Multi-Output system, as shown in Fig. 2, and expressed as:

$$
x_{k+1}=A x_{k}+B u_{k}+G \xi_{k}, \quad y_{k}=C x_{k},
$$

where $x_{k}$ is the state, $u_{k}$ is the control input, $y_{k}$ is the output, and $\xi_{k}$ is the process noise. The system is formulated to compensate the output disturbance, consisting of atmospheric turbulence, tracking a zero reference (a flat wavefront). More details on the formulation of optimal control in AO systems can be found in Konnik et al. ${ }^{10,11}$ 


\subsubsection{Formulation of Receding Horizon Control}

We use the standard state space formulation of $\mathrm{RHC}^{12}$ with quadratic cost function:

$$
V_{N_{p}, N_{c}}=\frac{1}{2} U^{T} \mathbb{H} U+U^{T} \mathbb{F} \hat{x}_{0},
$$

where $\hat{x}_{0}$ is the estimate of the current state obtained with a Kalman filter, $U=\left[u_{0}^{T}, u_{1}^{T}, \ldots u_{N_{c}-1}^{T}\right]^{T}$ is a vector of future controls, $N_{p}$ and $N_{c}$ are the state and control prediction horizons, respectively. The Hessian matrix $\mathbb{H}$ and matrices $\mathbb{F}, \Gamma$ and $\Omega$ are defined as:

$$
\mathbb{H} \triangleq \Gamma^{T} \mathcal{Q} \Gamma+\mathcal{R}, \quad \mathbb{F} \triangleq \Gamma^{T} \mathcal{Q} \Omega, \quad \text { where } \Gamma=\left[\begin{array}{ccc}
B & \ldots & 0 \\
A B & \cdots & 0 \\
\vdots & \ddots & \vdots \\
A^{N_{p}-1} B & \ldots & A^{N_{p}-N_{c}} B
\end{array}\right] \quad \text { and } \quad \Omega=\left[\begin{array}{c}
A \\
A^{2} \\
\vdots \\
A^{N_{p}}
\end{array}\right]
$$

here the matrices $\mathcal{Q}=\operatorname{diag}\left\{C^{T} Q C, \ldots P\right\}$ and $\mathcal{R}=\operatorname{diag}\{R, \ldots R\}$, and the matrices $Q=I, P=10^{-1} I$ and $R=10^{-9} I$ are tuning parameters. The goal for a Quadratic Programming (QP) algorithm is to minimise the cost function (6):

$$
\min _{U} \frac{1}{2} U^{T} \mathbb{H} U+U^{T} \mathbb{F} \hat{x}_{0} \quad \text { subject to } M \cdot U \leq \Lambda,
$$

where $M$ is the constraints matrix, and $\Lambda$ is the vector of maximum $U_{\max }$ and minimum $U_{\min }$ allowed values for the control input. The constrained optimisation problem (8) is solved online at each sampling instance.

\subsubsection{Formulation of Infinite Horizon Control}

Linear Quadratic Gaussian (LQG) control finds the state feedback gain off-line that minimises the cost function:

$$
J=\sum_{k=0}^{\infty}\left(\frac{1}{2} x^{T} Q x+\frac{1}{2} U^{T} R U\right)
$$

The matrix $Q$ is chosen as $Q=C^{T} C$, and the matrix $R$ is set either $R=10^{-10} I$ for a high-gain control or $R=10^{-2} I$ for a low-gain control. Solving the discrete-time algebraic Riccati equation (DARE):

$$
A^{T} \Omega_{K} A-A^{T} \Omega_{K} B\left(B^{T} \Omega_{K} B+R\right)^{-1} B^{T} \Omega_{K} A+Q=\Omega_{K}
$$

The solution of the DARE in (10) is the matrix $\Omega_{K}$, which is used for the state feedback gain calculation:

$$
K_{\text {gain }}=\left(B^{T} \Omega_{K} B+R\right)^{-1} B^{T} \Omega_{K} A
$$

where $Q=C^{T} C=\left[\begin{array}{ll}C_{\text {plant }} & C_{a t m}\end{array}\right]^{T}\left[\begin{array}{ll}C_{\text {plant }} & C_{a t m}\end{array}\right]$. The controller can be formulated as:

$$
U=-\operatorname{sat}\left[K_{\text {gain }} \hat{x}_{k}\right], \quad \operatorname{sat}(x)=\left\{\begin{array}{cc}
U_{\max } & \text { if } x \geq U_{\max } \\
x & \text { if } U_{\min } \leq x \leq U_{\max } \\
U_{\min } & \text { if } x \leq U_{\min }
\end{array}\right.
$$

where $U_{\max }$ and $U_{\min }$ are maximum and minimum allowed values for the control input, and $\hat{x}_{k}$ are the estimated states obtained by a Kalman filter.

\section{EVALUATION OF ATMOSPHERIC TURBULENCE REJECTION PERFORMANCE OF INFINITE AND RECEDING HORIZON CONTROL}

This section provides the results of end-to-end optical simulations for the unconstrained and constrained cases of closed-loop operation for Receding Horizon Control (RHC) and Linear Quadratic Gaussian (LQG) control. The performance was evaluated using the variance and the sum-of-squares of the residual turbulence. The RHC controller with the shortest prediction horizons $N_{p}=2, N_{c}=1$ in (7) and weighting matrices $P=10^{-1} I$ and $R=10^{-9} I$ was considered, and the LQG controller was considered as (explained further in Subsection 2.3.3): 
- high-gain $L Q G$ with the weighting matrix $R=10^{-10} I$ in $(10)$;

- low-gain $L Q G$ with the weighting matrix $R=10^{-2} I$ in (10).

The results of numerical simulations reported are for three cases of the inter-actuators coupling $\gamma$ :

- weak, with coupling degree $\gamma=0.10$, condition number of the Hessian matrix $\kappa(\mathbb{H}) \sim 10$;

- moderate, with coupling degree $\gamma=0.24$, condition number $\kappa(\mathbb{H}) \sim 400$;

- strong, with coupling degree $\gamma=0.30$, condition number $\kappa(\mathbb{H}) \sim 8000$.

All controllers were attempting to cancel the same atmospheric turbulence, which put them into the same conditions.

\subsection{Brief analysis of the optimal control problem for a deformable mirror}

The constrained case of operation of an AO system differs from the unconstrained operation, because the coupling between the actuators shifts the constrained optimum. This effect is illustrated in Subsection 2.1.1, which is important for interpreting the results of this contribution, and a discussion on the condition number and coupling degree is provided in Subsection 2.1.2.

\subsubsection{Influence of the inter-actuators coupling: an illustrative example}

We employ a simple example to illustrate the difference (and consequences) of the constrained operation of an adaptive optics system. Consider the following quadratic cost function:

$$
\min _{x} J(x)=\frac{1}{2} x^{T} \mathbb{H} x+x^{T} \mathbb{F}, \quad \mathbb{H}=\left[\begin{array}{ll}
1 & \xi \\
\xi & 1
\end{array}\right], \mathbb{F}=\left[\begin{array}{c}
0 \\
-4
\end{array}\right]
$$

where the parameter $\xi$ controls the condition number of the Hessian matrix $\mathbb{H}$. The goal of a controller is to find the optimal solution that minimises the quadratic objective function $J(x)$.

The main parameter that has a significant impact on the optimisation problem is the condition number of the Hessian matrix $\mathbb{H}$ defined as $\kappa(\mathbb{H})=\frac{\sigma_{\max }(\mathbb{H})}{\sigma_{\min }(\mathbb{H I})}$, where $\sigma_{\max }(\mathbb{H})$ and $\sigma_{\min }(\mathbb{H})$ are the maximal and minimal singular values. ${ }^{13}$ A matrix with a low condition number $\kappa(\mathbb{H})$ is said to be well-conditioned, and the contours of the cost function in (13) are isotropic (see Fig. 3(a)). This is the decoupled case, when there is no coupling between the actuators in a DM (e.g., in MEMS mirrors), and the solution of the constrained optimisation problem can be simply found by clipping the unconstrained solution.

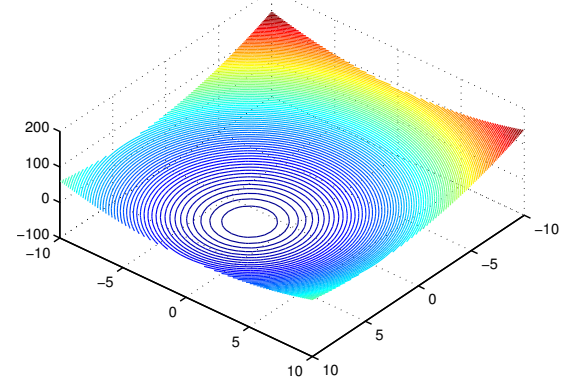

(a)

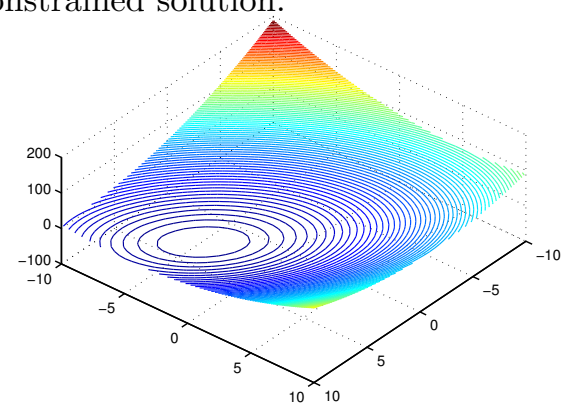

(b)

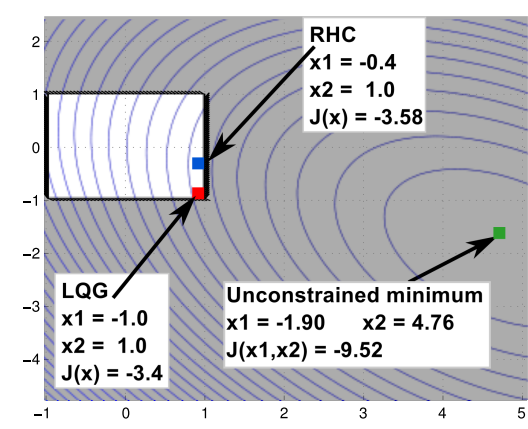

(c)

Figure 3. Contours of the cost function in (13): (a) the unconstrained case, no coupling between the actuators $(\xi=0)$; (b) the unconstrained case, strong coupling between the actuators $(\xi=0.4)$; (c) the constrained problem for the case of strong inter-actuators coupling $(\xi=0.4)$ and constraints $-1 \leq x_{1} \leq 1,-1 \leq x_{2} \leq 1$.

The inter-actuators coupling increases the condition number $\kappa(\mathbb{H})$ of the Hessian matrix, rendering the problem ill-conditioned (see also Subsection 2.1.2 and Fig. 4) and elongating the contours of the cost function in (13), as seen in Fig. 3(b). This shifts the constrained optimum and makes a simple clipping of control signals suboptimal, leading to unforeseen consequences discussed further in Subsections 2.3.2-2.3.3. Since continuous DMs usually have a relatively strong coupling ${ }^{14}$ between the actuators (up to 20-30\%), the problem of constrained operation in $\mathrm{AO}$ systems deserves attention. 
Consider imposing the following constraints on the variables in (13): $-1 \leq x_{1} \leq 1,-1 \leq x_{2} \leq 1$, rendering the optimisation problem constrained (see Fig. 3(c)). The LQG calculates the constrained solution via saturation (clipping) of the unconstrained minimum, giving the answer $x_{c}^{L Q R}=(-1.0,1.0)$ and the minimum of the cost function $\min J_{c}^{L Q R}\left(x_{1}, x_{2}\right)=-3.40$ (see Fig. 3(c)). However, the true constrained minimum of the objective function found by an optimisation algorithm is $x_{c}^{R H C}=(-0.4,1.0)$ that corresponds to $\min J_{c}^{R H C}\left(x_{1}, x_{2}\right)=-3.58$. This illustrates the point of this article: while the discrepancy of the cost function may seem small, the difference in the control input can be substantial, which may lead to unforeseen consequences.

\subsubsection{Growth of the coupling degree and variance of the dynamics increases the condition number thus shifting the constrained optimum}

The inter-actuators coupling in a DM is reflected in the condition number of the Hessian matrix $\kappa(\mathbb{H})$ defined in (7). If the deformable mirror is ideal with identical actuators, all of which have exactly the same dynamics, the only contributor to the condition number is the coupling degree $\gamma$. In this case the condition number should not be large. Indeed, as one can see from Fig. 4(a) marked by $-\bullet-$, the condition number of the Hessian matrix is $\kappa(\mathbb{H})<10^{3}$ even for a large coupling of $\gamma=0.35$.

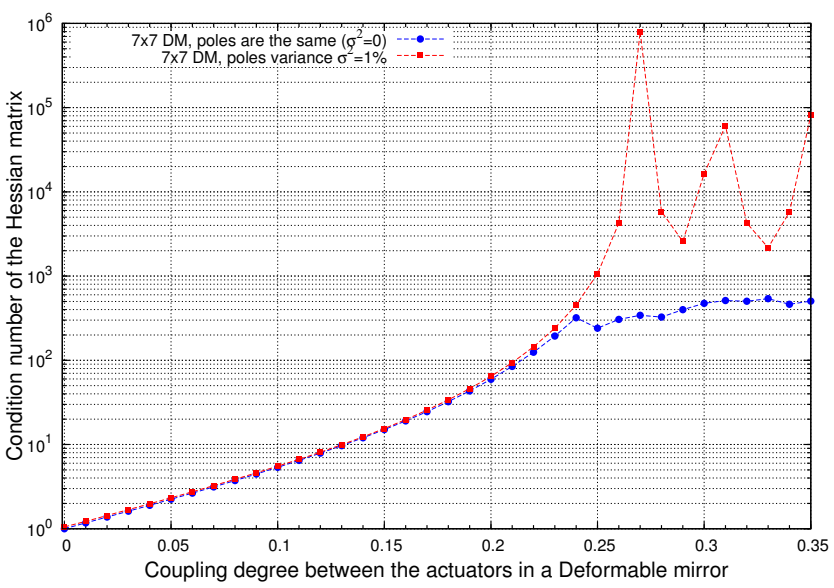

(a)

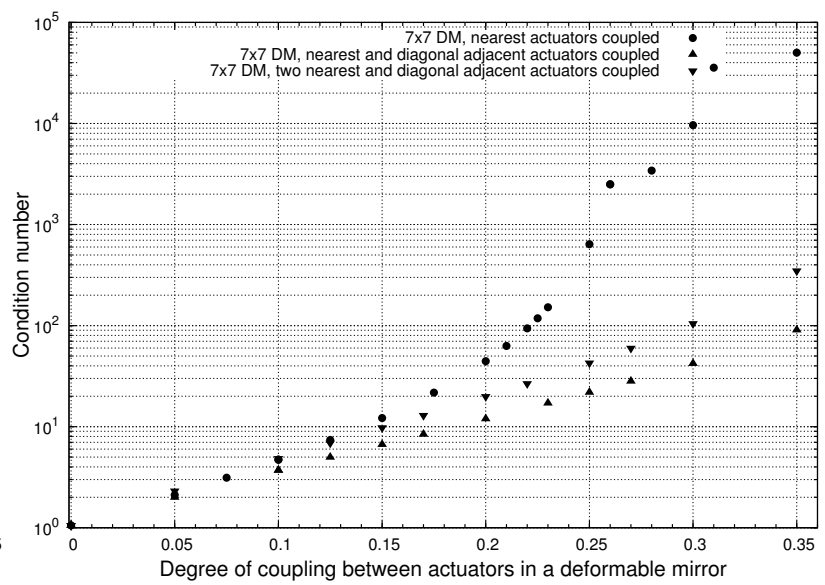

(b)

Figure 4. Condition number of the Hessian matrix for a $7 \times 7$ actuators DM: (a) dependence on the variability of actuators dynamics. (b) dependence on different types of coupling: four nearest actuators, marked by $-\bullet-$, four nearest and four diagonally adjacent, marked by $-\boldsymbol{\nabla}-$, and two groups of nearest actuators plus diagonally adjacent, marked by $-\boldsymbol{\Delta}-$.

The variance in the dynamics of actuators in a DM is another contributor to the growth of the condition number of the Hessian matrix $\kappa(\mathbb{H})$. As one can see from Fig. 4(a) marked by - - -, the condition number can reach $\kappa(\mathbb{H}) \sim 10^{6}$. The condition number in the case when there is coupling between eight nearest neighbour actuators (four nearest and four diagonally adjacent) is shown in Fig. 4(b).

\subsection{The Unconstrained Case: the control signal is within the range of actuators stroke}

In the unconstrained case, the control signal remains within the safe operation margins. Therefore, the residual disturbance should be similar because both RHC and LQG solve similar optimisation problems in (6) and (9).

Indeed, the performance of high-gain LQG and RHC is the same in terms of both sum-of-squares (see Fig. 5(b)) and the variance of the residual disturbance (see Fig. 5(a)). The relatively low compensational performance is explained by the fact that we estimated the dynamics of the atmospheric turbulence from the data via a stochastic system identification algorithm*. However, we use the same values of the atmospheric turbulence and the same state space matrices for both controllers to put them into the same conditions.

\footnotetext{
${ }^{*}$ Despite a rather large (about 20000 samples) dataset, the values in the state space matrices may be biased, and further configuration of the stochastic system identification algorithms is needed.
} 


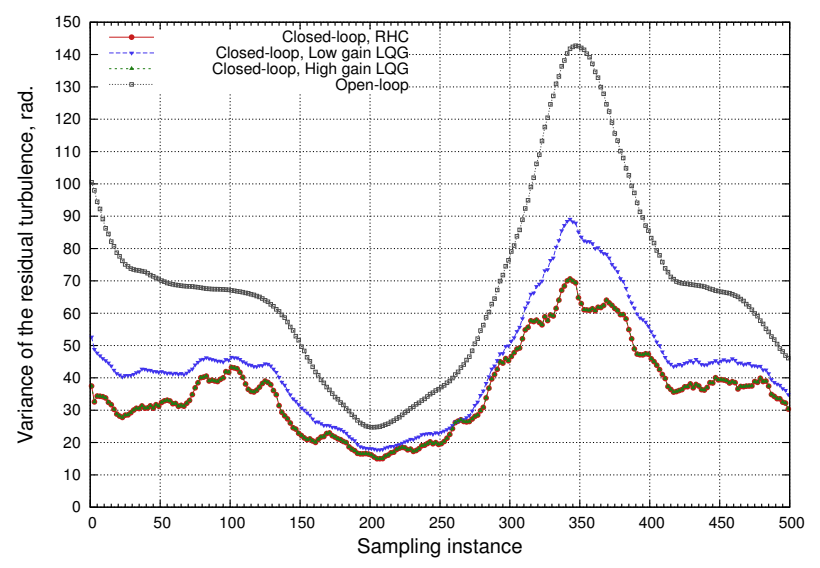

(a)

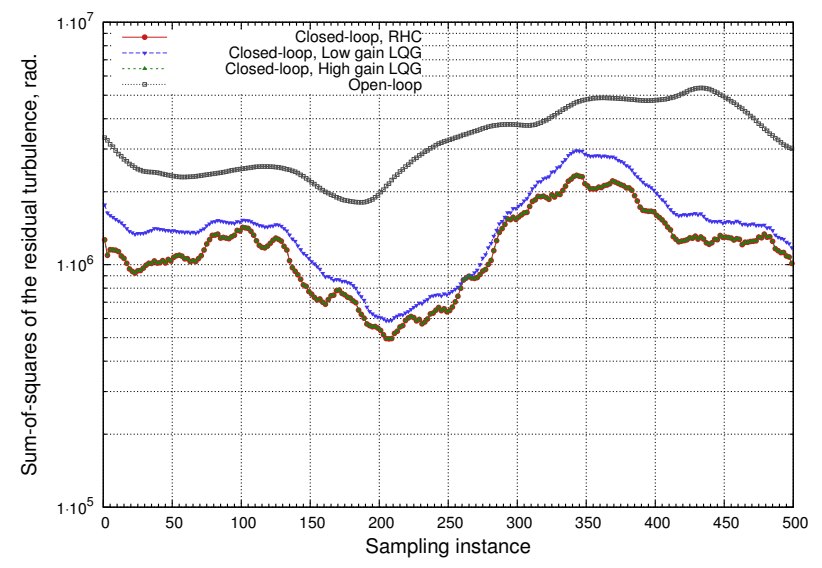

(b)

Figure 5. Atmospheric turbulence rejection performance for the LQG and RHC controllers in the unconstrained case: (a) Variance of the residuals; (b) Sum of squares of the residuals. The case of coupling between the four nearest actuators in the $8 \times 8 \mathrm{DM}$ is shown for $1000 \mathrm{~Hz}$ sampling rate.

\subsection{The Constrained Case: the control signal exceeds the range of actuators stroke}

In the unconstrained case both RHC and LQG controllers give a similar disturbance rejection performance (see Fig. 5). This is expected, because both RHC and LQG solve a similar optimisation problem. The constrained case of AO operation is different, because the coupling between the actuators increases the condition number (compare Fig. 3(a) and Fig. 3(b)). We therefore simulate the constrained closed-loop operation of an AO system for a different strength of coupling between the DM actuators: the cases of weak, moderate and strong interactuators coupling for LQG and RHC are considered in Subsections 2.3.1, 2.3.2, and 2.3.3, respectively.

\subsubsection{Results for a weak coupling between actuators}

We consider a DM with a weak coupling $(\gamma=0.10)$ between four nearest actuators only. This coupling degree corresponds to the condition number of the Hessian matrix $\kappa(\mathbb{H}) \sim 10$, which means that the matrix is wellconditioned. In this case we should not expect much benefits in solving the constrained optimisation problem by RHC compared to LQG, since the clipped unconstrained solution is close to the constrained optimum.

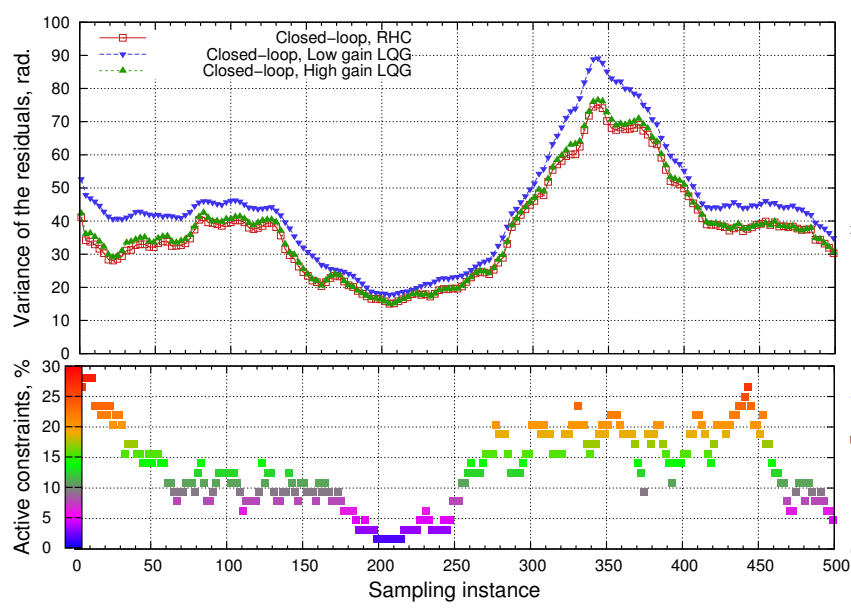

(a)

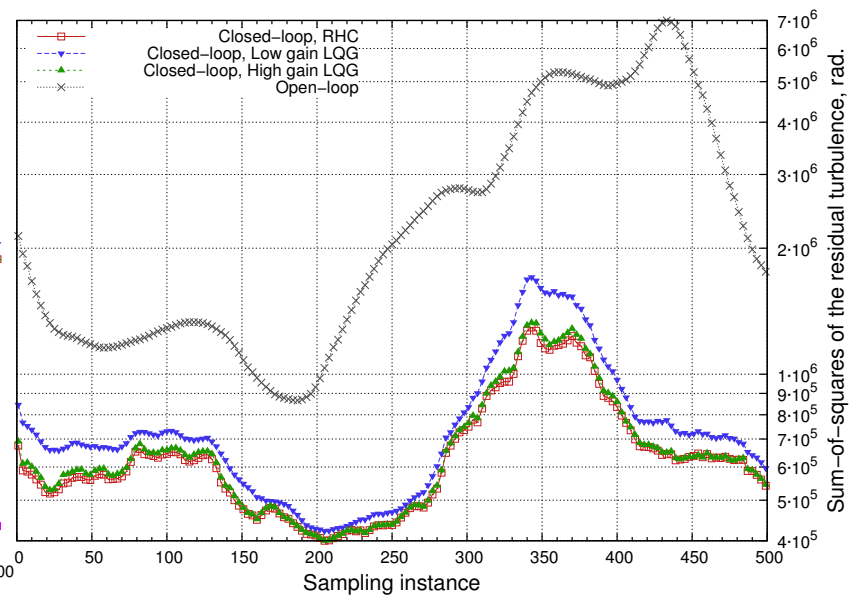

(b)

Figure 6. Atmospheric turbulence rejection performance for LQG and RHC as measured via: (a) variance of the residuals depending on the percentage of active constraints; (b) sum of squares of the residuals. The case of weak coupling (coupling degree $\gamma=0.10$, condition number $\kappa(\mathbb{H}) \sim 10$ ) for an $8 \times 8$ actuators DM is shown. 
Indeed, the results in Fig. 6 indicate that the atmospheric turbulence rejection performance is similar for LQG and RHC, as expected. One can notice from Fig. 6(b) that RHC provides 2-5\% less variance of the residual turbulence than LQG in the case when only few (5-10\%) constraints are active. In the case of many active constraints (e.g., in sampling instances 0-30 and 350-400 in Fig. 6(a)) the performance of high-gain LQG drops by 3-7\% compared to RHC. The same effect can be seen in Fig. 6(b) for the sum-of-squares of the residuals.

\subsubsection{Results for a moderate coupling between actuators}

The increased coupling between the actuators leads to a deteriorated performance of LQG in the constrained case. The high-gain LQG control has 10-20\% more variance of the residual turbulence than RHC when there are few constraints active, and up to $30 \%$ more variance of the residuals in the case when there are many (15-25\%) active constraints (see Fig. 7(a), sampling instances 0-100 and 350-400). The low-gain LQG control produces less peaking (see Fig. 8) in the control signal, but it under-actuates the DM and thus is 10-20\% worse than RHC in terms of variance of the residuals. This should not be surprising, because the constrained optimisation problem has an increased condition number of the Hessian matrix $\kappa(\mathbb{H}) \sim 400$ due to the inter-actuators coupling, which in turn shifts the constrained optimum and makes a simple clipping of the control signals suboptimal.

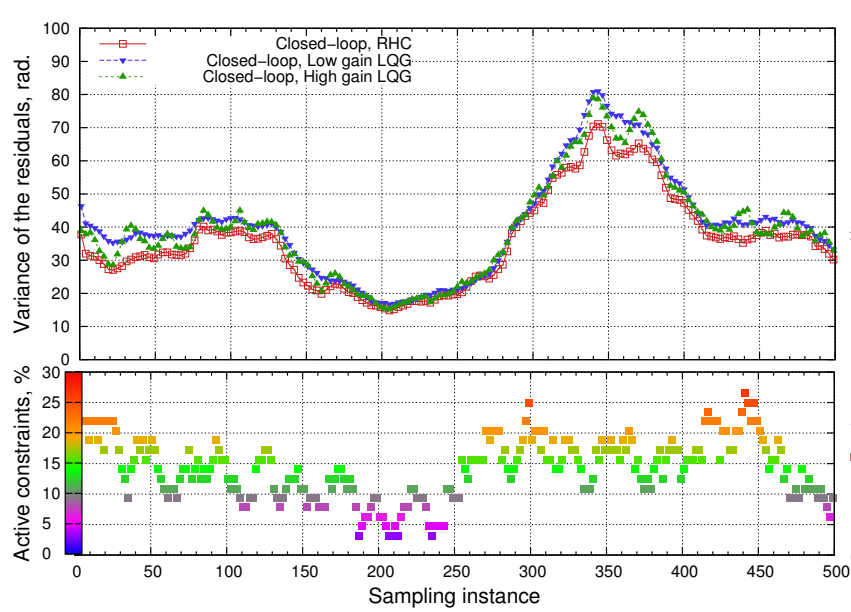

(a)

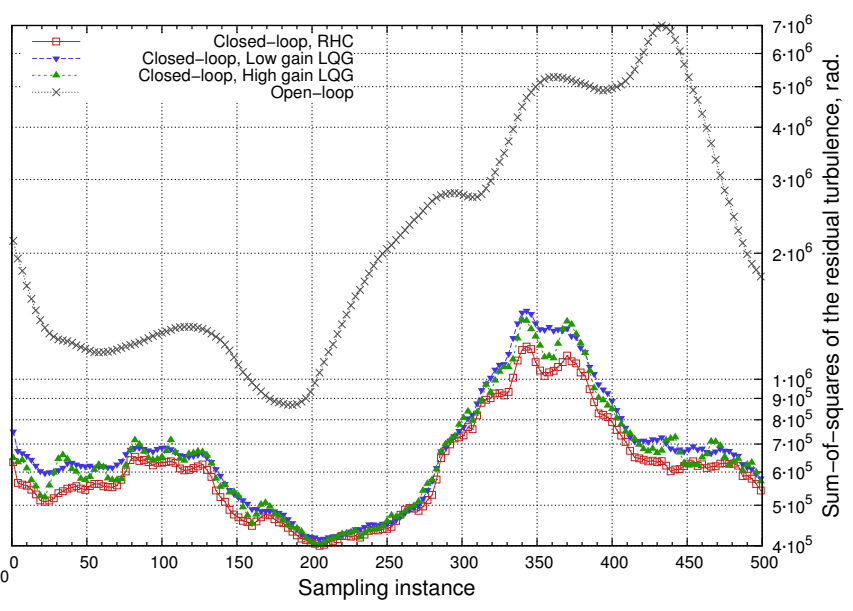

(b)

Figure 7. Atmospheric turbulence rejection performance for LQG and RHC as measured via: (a) variance of the residuals depending on the percentage of active constraints; (b) sum of squares of the residuals. The case of moderate coupling (the coupling degree $\gamma=0.24$, the condition number $\kappa(\mathbb{H}) \sim 400$ ) for an $8 \times 8$ actuators DM is shown.

Moreover, in this case the low-gain $L Q G$ (marked by $-\boldsymbol{\nabla}$ - in Fig. 7) with the weighting matrix set to $R=10^{-2} I$ in (10) sometimes outperforms the high-gain LQG (marked by - $\Delta$ - in Fig. 7 ). The peaking of the control signal is the main reason why a high-gain LQG controller (marked by $-\mathbf{\Delta}-$ in Fig. 8 ) is outperformed by RHC (marked by $-\square-$ in Fig. 8) in the constrained case and moderate coupling degree of $\gamma=0.24$.

One can make the LQG controller less aggressive by lowering the gains via the weighting matrix (using $R=10^{-2} I$ instead of $R=10^{-10} I$ as in the high-gain case) in (10). However, low-gain LQG (marked by - $\boldsymbol{\nabla}-$ in Fig. 8) does not use the full actuators authority and therefore provides worse performance compared to RHC (marked by - - -). This trend will be more pronounced as the inter-actuators coupling grows larger (see also Subsection 2.3.3 and Table 1 of Subsection 3.1). 


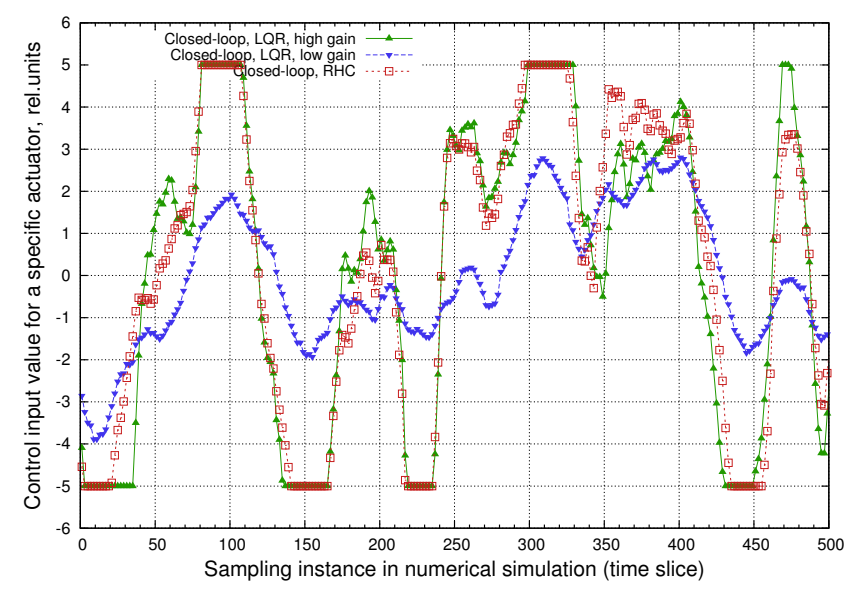

(a)

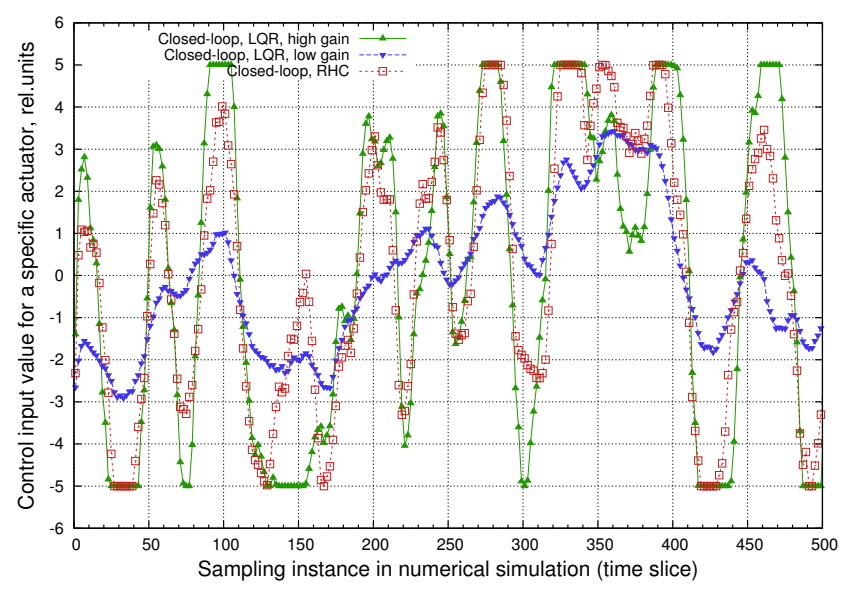

(b)

Figure 8. Comparison of the LQG and RHC control inputs for the actuators in the $8 \times 8$ DM for the same atmospheric turbulence: (a) control signal for the actuator 21; (b) control signal for the actuator 28. The case of moderate coupling (coupling degree $\gamma=0.24$, condition number $\kappa(\mathbb{H}) \sim 400$ ) is shown.

\subsubsection{Results for a strong coupling between actuators}

When the actuators in a deformable mirror have a strong coupling, which in our simulations is described by the coupling degree $\gamma=0.30$, the corresponding condition number of the Hessian matrix $\kappa(\mathbb{H}) \sim 8000$ renders the constrained optimisation problem ill-conditioned. Consequently, the constrained optimum is being shifted from the clipped unconstrained solution (which is the saturated LQG control) even further, leading to further deterioration of turbulence rejection performance of LQG compared to RHC, as seen in Fig. 9(a).

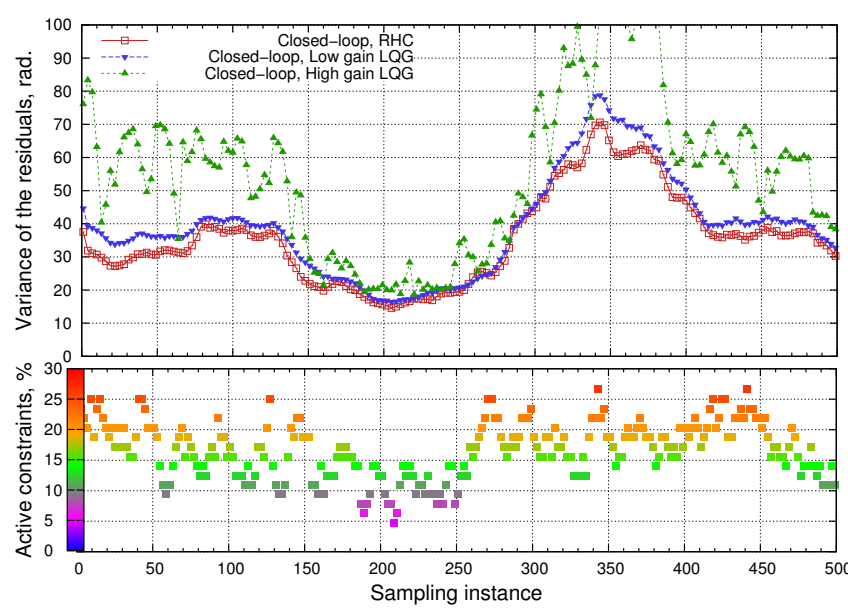

(a)

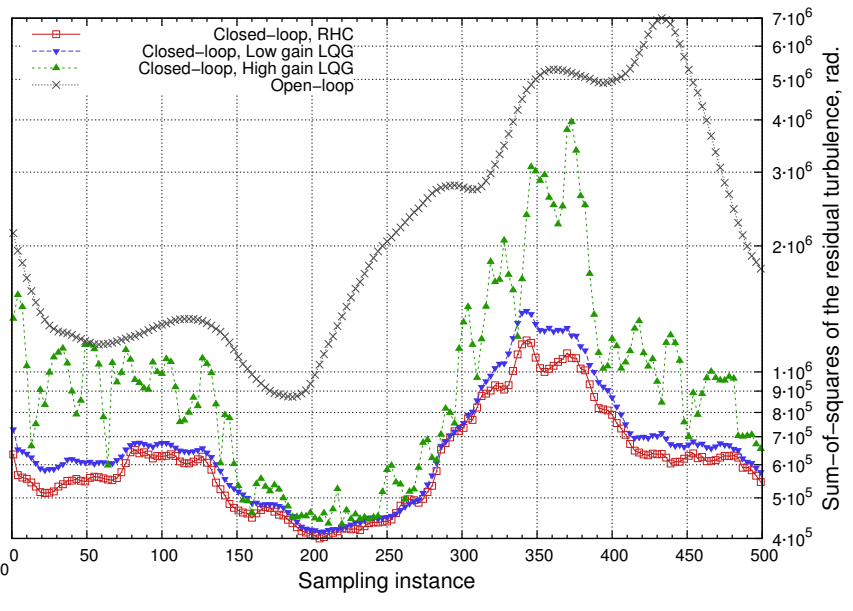

(b)

Figure 9. Atmospheric turbulence rejection performance for LQG and RHC as measured via: (a) variance of the residuals depending on the percentage of active constraints; (b) sum of squares of the residuals. The case of strong coupling (coupling degree $\gamma=0.30$, condition number $\kappa(\mathbb{H}) \sim 8000$ ) is shown.

When only a few (5-10\%) constraints are active (see sampling instances 180-250 in Fig. 9(a)), RHC has $30-45 \%$ lower variance of the residuals than high-gain LQG. The case when there are many active constraints is expectedly worse: while this is a rare case of operation for a typical adaptive optics system, the high-gain LQG control is sometimes only marginally better than an open-loop operation (marked by $-x-$ in Fig. 9(b)). There is a significant performance deterioration when there are many active constraints: one can see in Fig. 9 (a) that 
high-gain LQG has up to 100-150\% more variance of the uncompensated turbulence than RHC. This is because the high-gain LQG control (see Fig. 10 and also Fig. 11) is too aggressive for constrained operation, which causes peaking and abrupt changes in the control signal.

Such a loss of the turbulence rejection performance by LQG in the constrained operation mode of an adaptive optics system becomes more apparent from the plots of the control signals versus time presented in Fig. 10. One can see in Fig. 10(a) for actuator 21 the abrupt spikes of the control signal produced by a high-gain LQG (marked by $-\mathbf{\Delta}_{-}^{-}$), and at the same time a considerable under-actuation of a low-gain LQG (marked by $-\boldsymbol{\nabla}-$ ).

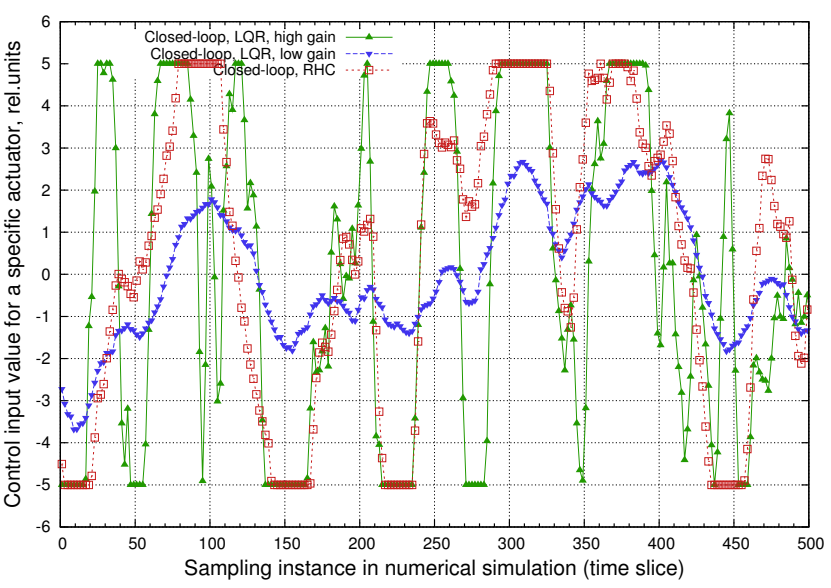

(a)

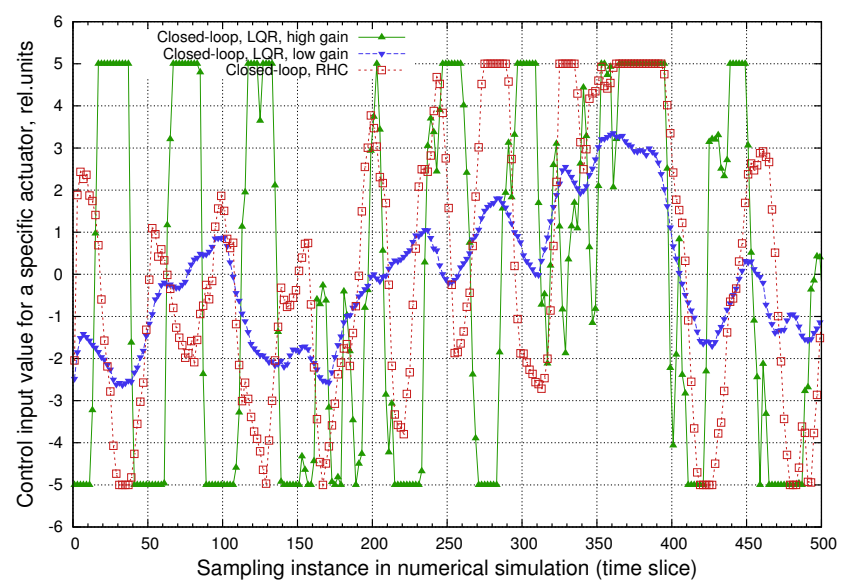

(b)

Figure 10. Comparison of the LQG and RHC control inputs for the actuators in the $8 \times 8$ DM for the same atmospheric turbulence: (a) control signal for the actuator 21; (b) control signal for the actuator 28. The case of strong coupling (coupling degree $\gamma=0.30$, condition number $\kappa(\mathbb{H}) \sim 8000$ ) is shown.

While lowering the gain by adjusting the weighting matrix $R$ in (10) might be considered as a work-around, this does not solve the problem: the low-gain LQG controller yields lower performance in both constrained and unconstrained cases, as seen in Fig. 5(a), where the performance of low-gain LQG (marked by $-\boldsymbol{\nabla}-$ ) drops by up to $30-40 \%$ compared to RHC (marked by $-\square-$ ). This is due to under-actuation and not using the full authority of the actuators, as one can also see from Fig. 11(b).

\section{PERFORMANCE COMPARISON OF LQG AND RHC STRATEGIES IN THE CONSTRAINED OPERATION OF ADAPTIVE OPTICS SYSTEMS}

In this section we summarise the performance comparison of LQG and RHC for an adaptive optics system in terms of atmospheric turbulence rejection and computational time. We discuss in Subsection 3.1 the benefits of low- and high-gain modes of LQG controller and why RHC may be a better choice for AO control. We also provide a brief comparison of LQG and RHC in terms of computational time in Subsection 3.2.

\subsection{Comparison of atmospheric turbulence rejection performance of LQG and RHC}

We consider two modes of operation of an adaptive optics system - the unconstrained (which is the main mode) and the constrained mode. Using either the variance of the residual (uncompensated) turbulence or the sumof-squares of the residuals, we compare the atmospheric turbulence rejection performance for LQG (low-gain and high-gain) and RHC. The reason for high-gain LQG is that we obviously want to compensate as much atmospheric turbulence as possible. This is a reasonable choice in the unconstrained mode, when the highgain LQG controller gives similar performance as RHC (see Fig. 5). Even when the actuators in the DM are weakly coupled, the performance of LQG is only marginally worse than the performance of RHC (see Fig. 6).

One may therefore ask the question: Why use RHC or low-gain $L Q G$ if the high-gain LQG controller does a good job in turbulence compensation? To answer this question, we have to recall that many deformable mirrors 
have actuators that are spaced close together to provide a high-accuracy correction. For example, the DMs for the ELT-class telescopes ${ }^{14}$ may require tens of thousands of actuators with inter-actuator spacing $200 \mu \mathrm{m}-1 \mathrm{~mm}$ and coupling up to $20-30 \%$. Such a degree of coupling (more than 20\%) shifts the constrained optimum far enough from the clipped unconstrained optimum for saturated LQG control to start producing peaks and abrupt changes in the control signal.

A large condition number elongates the cost function contours (see Fig. 3 and the discussion in Subsection 2.1.1) and shifts the constrained optimum further away from the clipped/saturated unconstrained solution of the optimisation problem. This results in more and more suboptimal control signals being generated by a clipped high-gain LQG controller, to the point where such a control begins to produce peaking (see Fig. 10) and, consequently, a substantial (by 100-150\% in variance of the residuals) loss of atmospheric turbulence rejection performance of the high-gain LQG (see Fig. 11(a), marked by - -- ) compared to RHC performance.

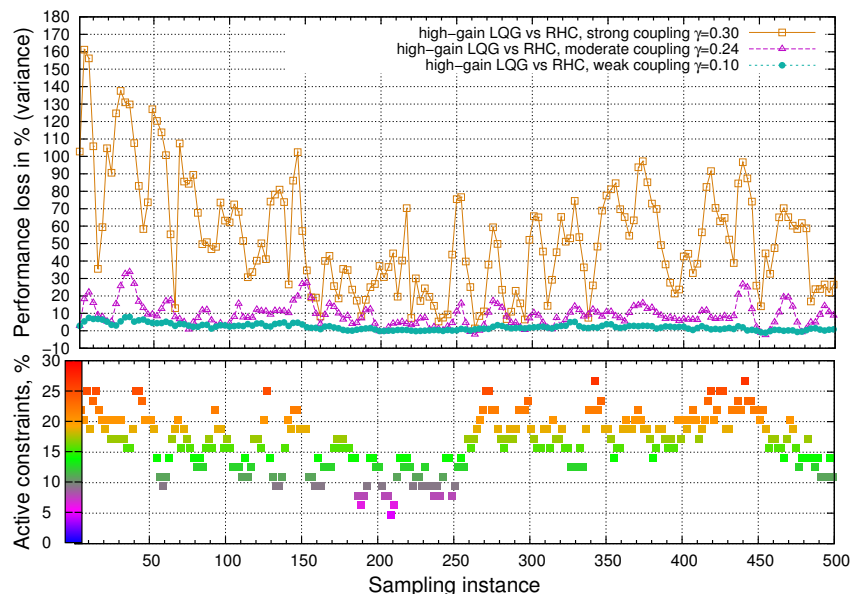

(a)

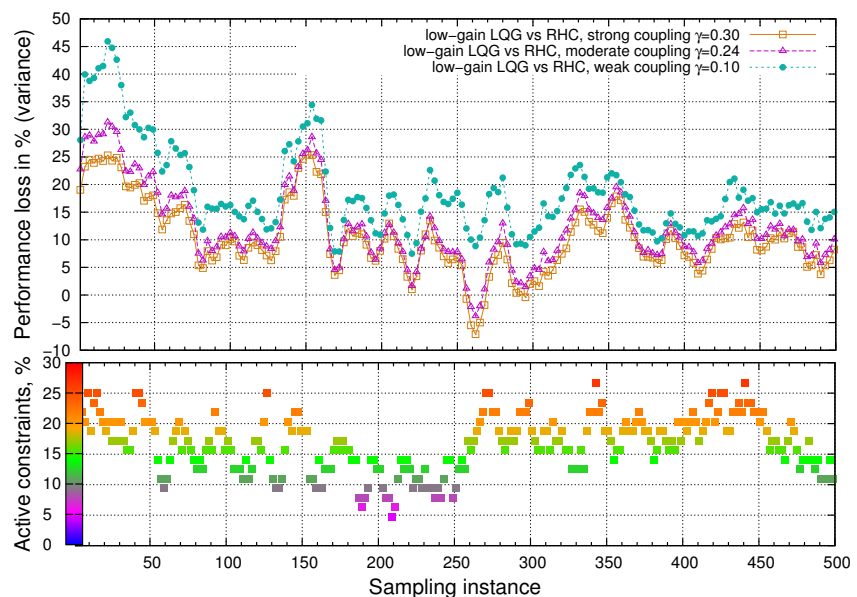

(b)

Figure 11. Deterioration of the turbulence rejection performance of LQG in percents from the RHC performance: (a) for the high-gain LQG; (b) for the low-gain LQG controller.

Lowering the gain for the LQG controller via weighting matrices (setting $R=10^{-2} I$ in $(10)$ ) improves the overall performance by reducing the peaking of the control signals (see Fig. 11(b), marked by - $\square-$ ). While low-gain LQG has a better performance (closest to RHC according to Fig. 11(b)) than high-gain LQG in this case, this comes at the price of under-actuating the actuators of the deformable mirror. The RHC control, on the other hand, provides better control in both unconstrained and constrained cases, since RHC solves the constrained QP problem online at each sampling instance. The quantitative advantage of RHC over LQG is summarised in Table 1.

Table 1. The advantage of Receding Horizon Control over Linear Quadratic Gaussian: a comparison of turbulence rejection performance (i.e., RHC is \% better than LQG in variance of the residual atmospheric turbulence).

\begin{tabular}{|c|c|c|c|c|c|c|}
\hline \multirow[b]{2}{*}{ Controller $\gtrsim$} & \multicolumn{3}{|c|}{ Few $(\mathbf{5 - 1 0 \%})$ active constraints } & \multicolumn{3}{|c|}{ Many (15-25\%) active constraints } \\
\hline & $\begin{array}{c}\text { weak } \\
\gamma=0.10 \\
\kappa(\mathbb{H}) \sim 10\end{array}$ & $\begin{array}{c}\text { moderate } \\
\gamma=0.24 \\
\kappa(\mathbb{H}) \sim 400\end{array}$ & $\begin{array}{c}\text { strong } \\
\gamma=0.30 \\
\kappa(\mathbb{H}) \sim 8000\end{array}$ & $\begin{array}{c}\text { weak } \\
\gamma=0.10 \\
\kappa(\mathbb{H}) \sim 10\end{array}$ & $\begin{array}{c}\text { moderate } \\
\gamma=0.24 \\
\kappa(\mathbb{H}) \sim 400\end{array}$ & $\begin{array}{c}\text { strong } \\
\gamma=0.30 \\
\kappa(\mathbb{H}) \sim 8000\end{array}$ \\
\hline ain LQG & $2-5 \%$ & $10-25 \%$ & $20-40 \%$ & $3-7 \%$ & $15-30 \%$ & $100-150 \%$ \\
\hline Low-gain LQG & $5-20 \%$ & $5-15 \%$ & $5-10 \%$ & $30-50 \%$ & $15-30 \%$ & $10-20 \%$ \\
\hline
\end{tabular}

\subsection{Computational performance comparison of LQG and RHC}

One may expect that solving an optimisation problem online for RHC is prohibitively expensive in terms of computational time. After all, the LQG controller uses a matrix of static gains to produce the control signal, 
and therefore is extremely fast: it takes $25-30 \mu$ s to compute the control inputs for a $7 \times 7$ actuators DM, and 60-70 $\mu$ s for a $10 \times 10$ actuators DM (see Fig. $12(\mathrm{a})$ ). However, when the actuators in a DM have fast dynamics, the shortest prediction horizons $N_{p}=2, N_{c}=1$ are sufficient for a good turbulence rejection performance in RHC. Furthermore, astronomical adaptive optics systems have inherently fast sampling rates, which can be exploited by hot-started QP algorithms. That is, online optimisation algorithms can quickly locate the new optimum within few iterations in the case of hot-start, even when many constraints are active.

It is not surprising that under the aforementioned conditions the Receding Horizon Controller with hot-started Range-Space Primal Active Set QP is almost as fast as the LQG controller, as seen in Fig. 12(a). The RHC is slightly slower than LQG, especially when more constraints are active (corresponding to a strong turbulence). For example, LQG takes $60-65 \mu$ s for a $10 \times 10$ actuators DM, while RHC takes from $65-70 \mu$ s to $90-120 \mu$ s. This is because two or more iterations are necessary to find the new optimum, which can also be seen in Fig. 12(b) for the growth rate of the computational time for RHC and LQG.

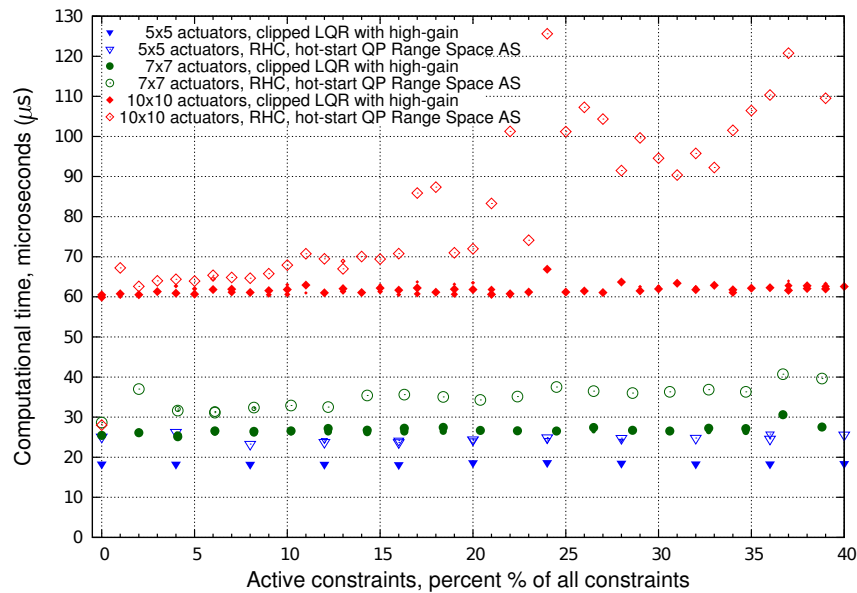

(a)

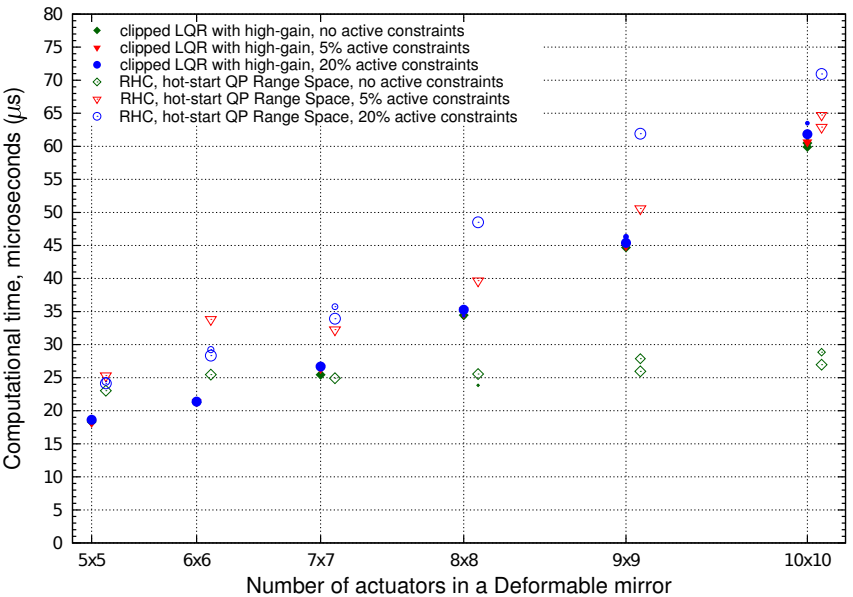

(b)

Figure 12. Comparison of computational time for LQG and RHC controllers: (a) time versus percentage of active constraints; (b) growth rate of computational time versus the number of actuators in the model of a DM.

Further developments and customisation of the QP algorithms can improve the performance of RHC. For example, Parametric Active Set methods (and especially homotopy-based ${ }^{15}$ algorithms like qpOASES), have an almost constant computational time with the percentage of active constraints. This is a highly desirable property of a QP algorithm, because it makes the behaviour of the solver predictable in terms of the computational time. The efficiency of a hot-start is crucial: the hot-start of QP algorithms in Receding Horizon Control for Adaptive Optics systems is a key technique that allows achieving extremely fast computation times.

\section{SUMMARY AND CONCLUSIONS}

This contribution reports performance evaluation results for Receding Horizon Control (RHC) and Linear Quadratic Gaussian (LQG) control in terms of the computational time and the atmospheric turbulence rejection.

Summary of atmospheric turbulence rejection performance. The benefits of RHC grow as the condition number of the Hessian matrix increases when an adaptive optics system works in constrained mode. For the example of $8 \times 8$ actuators DM, even in the case of weak coupling $(\gamma=0.10, \kappa(\mathbb{H})=10)$, the variance of residuals for RHC is 3-7\% less than for high-gain LQG. In the case of moderate coupling $(\gamma=0.24)$ RHC has $10-20 \%$ less variance of residual turbulence than high-gain LQG control when there are few constraints active, and up to $30 \%$ less variance when there are many (15-25\%) active constraints. When there is a strong coupling between the actuators $(\gamma=0.30)$ and the optimisation problem becomes not well-conditioned $(\kappa(\mathbb{H})=8000)$, the performance of high-gain LQG deteriorates substantially: the variance of the residuals is $20-40 \%$ more compared to RHC in case of few active constraints and up to 100-150\% when there are many active constraints. In this case, low-gain LQG is preferable, since it has only $5-15 \%$ more variance of uncompensated residuals. 
Summary of computational performance evaluation. When the sampling rate is fast and the dynamics of the atmospheric turbulence is slow, the hot-start of QP algorithms in Receding Horizon Control for Adaptive Optics systems is a key technique that allows to achieve extremely fast computation times for a constrained control. For the shortest prediction horizons $N_{p}=2, N_{c}=1$, which are sufficient for a good turbulence rejection performance of RHC, the computational times are comparable for LQG and RHC. For an example of a $10 \times 10$ actuators DM, the LQG controller takes $60-65 \mu \mathrm{s}$, while RHC takes from $65-70 \mu$ s to $90-120 \mu \mathrm{s}$.

Receding Horizon Control: pros and cons. The slower the dynamics of the actuators, the longer the prediction horizons must be (e.g., membrane mirrors ${ }^{16}$ may require $N_{p}=20$ ), which makes a constrained operation difficult. For certain types of mirrors, such as MEMS with decoupled actuators, RHC is unlikely to provide noticeable performance advantages (apart from easier implementation).

On the other hand, RHC is more predictable (no peaking and less numerical problems) and is easier to implement ${ }^{\dagger}$, which is important for astronomical adaptive optics. Large-scale ELT-size RHC is possible via distributed optimisation framework. The benefits of RHC grows as the condition number of the Hessian matrix increases due to inter-actuators coupling, allowing the use of adaptive optics in stronger turbulence and attaining the full compensation potential of deformable mirrors.

\section{REFERENCES}

[1] Herman, B. and Strugala, L., "Method for inclusion of low-frequency contributions in numerical representation of atmospheric turbulence," in [Proceedings of SPIE], 1221, 183 (1990).

[2] Sedmak, G., "Performance analysis of and compensation for aspect-ratio effects of fast-Fourier-transformbased simulations of large atmospheric wave fronts," Applied optics 37(21), 4605-4613 (1998).

[3] Schmidt, J. D., [Numerical Simulation of Optical Wave Propagation, With Examples in Matlab], Society of Photo-Optical Instrumentation Engineers (2010).

[4] Assémat, F., Wilson, R., and Gendron, E., "Method for simulating infinitely long and non stationary phase screens with optimized memory storage," Optics express 14(3), 988-999 (2006).

[5] Peter van Overschee and Bart de Moor, [Subspace Identification for Linear Systems: Theory - Implementation - Applications], Springer (1996).

[6] Thomas, S., Fusco, T., Tokovinin, A., Nicolle, M., Michau, V., and Rousset, G., "Comparison of centroid computation algorithms in a shack-hartmann sensor," Monthly Notices of the Royal Astronomical Society 371(1), 323-336 (2006).

[7] Poyneer, L., Troy, M., Macintosh, B., and Gavel, D., "Experimental validation of Fourier-transform wavefront reconstruction at the Palomar Observatory," Optics Letters 28(10), 798-800 (2003).

[8] Franke, R., "Scattered data interpolation: tests of some methods," Mathematics of computation 38(157), 181-200 (1982).

[9] Buhmann, M. D., [Radial basis functions: theory and implementations], Cambridge University Press (2003).

[10] Konnik, M. V., Online Constrained Receding Horizon Control for Astronomical Adaptive Optics, PhD thesis, School of Electrical Engineering and Computer Science, the University of Newcastle, Australia (2013).

[11] Mikhail V. Konnik, José De Doná, and James Stuart Welsh, "On application of constrained receding horizon control in astronomical adaptive optics," in [Proceedings of SPIE Astronomical Telescopes + Instrumentation - Adaptive Optics Systems III], 8447-110 (2012).

[12] Graham Clifford Goodwin, Maria Marta Seron, and José De Doná, [Constrained Control and Estimation: An Optimisation Approach], Springer, Berlin (2005).

[13] Hogben, L., [Handbook of linear algebra], CRC Press (2007).

[14] Foy, R. and Foy, F. C., "Optics in astrophysics," in [Proceedings of the NATO Advanced Study Institute on Optics in Astrophysics, 16-28 September, Cargèse, France], Nato Science Series II 198, Springer (2005).

[15] Best, M. J., "An algorithm for the solution of the parametric quadratic programming problem," Applied Mathematics and Parallel Computing, 57-76 (1996).

[16] Tesch, J., Gibson, S., and Verhaegen, M., "Receding-horizon adaptive control of aero-optical wavefronts," Optical Engineering 52(7), 071406-071406 (2013).

\footnotetext{
${ }^{\dagger}$ A typical QP algorithm for RHC amounts to 500-1000 lines of C-code, unlike DARE solvers for LQG that often require eigenvalue decomposition algorithms and other advanced numerical methods.
} 